\title{
Safe and Sustained Expression of Human Iduronidase After Intrathecal Administration of Adeno-Associated Virus Serotype 9 in Infant Rhesus Monkeys
}

\author{
Juliette Hordeaux, ${ }^{1}$ Christian Hinderer, ${ }^{1}$ Elizabeth L. Buza, Jean-Pierre Louboutin, ${ }^{2}$ \\ Tahsin Jahan, ${ }^{1}$ Peter Bell, ${ }^{1}$ Jessica A. Chichester, ${ }^{1}$ Alice F. Tarantal, ${ }^{3}$ and James M. Wilson ${ }^{1, *}$ \\ ${ }^{1}$ Gene Therapy Program, Department of Medicine, University of Pennsylvania, Perelman School of Medicine, Philadelphia, Pennsylvania; \\ ${ }^{2}$ Section of Anatomy, Department of Basic Medical Sciences, University of West Indies, Kingston, Jamaica; ${ }^{3}$ Center for Fetal Monkey \\ Gene Transfer for Heart, Lung, and Blood Diseases, Departments of Pediatrics and Cell Biology and Human Anatomy, School of Medicine, \\ and California National Primate Research Center, University of California, Davis, California.
}

Many neuropathic diseases cause early, irreversible neurologic deterioration, which warrants therapeutic intervention during the first months of life. In the case of mucopolysaccharidosis type I, a recessive lysosomal storage disorder that results from a deficiency of the lysosomal enzyme $\alpha$-l-iduronidase (IDUA), one of the most promising treatment approaches is to restore enzyme expression through gene therapy. Specifically, administering pantropic adeno-associated virus (AAV) encoding IDUA into the cerebrospinal fluid (CSF) via suboccipital administration has demonstrated remarkable efficacy in large animals. Preclinical safety studies conducted in adult nonhuman primates supported a positive risk-benefit profile of the procedure while highlighting potential subclinical toxicity to primary sensory neurons located in the dorsal root ganglia (DRG). This study investigated the long-term performance of intrathecal cervical AAV serotype 9 gene transfer of human IDUA administered to 1-month-old rhesus monkeys $(N=4)$ with half of the animals tolerized to the human transgene at birth via systemic administration of an AAV serotype 8 vector expressing human IDUA from the liver. Sustained expression of the transgene for almost 4 years is reported in all animals. Transduced cells were primarily pyramidal neurons in the cortex and hippocampus, Purkinje cells in the cerebellum, lower motor neurons, and DRG neurons. Both tolerized and non-tolerized animals were robust and maintained transgene expression as measured by immunohistochemical analysis of brain tissue. However, the presence of antibodies in the non-tolerized animals led to a loss of measurable levels of secreted enzyme in the CSF. These results support the safety and efficiency of treating neonatal rhesus monkeys with AAV serotype 9 gene therapy delivered into the CSF.

Keywords: AAV9, intrathecal, MPS I, infant, rhesus monkey

\section{INTRODUCTION}

MUCOPOLYSACCHARIDOSIS TYPE 1 (MPS I) is a rare recessive disease caused by mutations in the gene encoding $\alpha$-L-iduronidase (IDUA), a lysosomal enzyme required for degradation of glycosaminoglycans (GAGs). IDUA deficiency causes multi-systemic accumulation of GAGs and other metabolites, leading to broad and diverse clinical manifestations. Hepatosplenomegaly, bone deformities, corneal clouding, and cardiac insufficiency are the main peripheral manifestations of the disease. ${ }^{1,2}$ Patients affected with the severe form of MPS I, referred to as Hurler disease, develop lysosomal storage lesions in the central nervous system (CNS) with progressive cognitive impairment beginning in early childhood. ${ }^{1,3}$

Enzyme replacement therapy can improve many of the systemic manifestations of the disease. ${ }^{4-6}$ However, the CNS impairment remains unaltered due to the impermeability of the blood-brain barrier

* Correspondence: Dr. James M. Wilson, Gene Therapy Program, Perelman School of Medicine, University of Pennsylvania, 125 South 31 st Street, Suite 1200, Philadelphia, PA 19104. E-mail: wilsonjm@upenn.edu 
to delivered enzymes systemically. ${ }^{7}$ Hematopoietic stem-cell transplantation, when performed sufficiently early, can achieve enzyme delivery to the CNS through the migration of donor-derived macrophage progenitor cells into the brain. ${ }^{8}$ However, the conditioning regimen and the potential for graft-versus-host disease are associated with morbidity and mortality, and the kinetics of donor cell engraftment in the CNS is slow. ${ }^{9}$

A rapid and safe gene therapy strategy was developed in which the IDUA coding sequence expressed from a strong ubiquitous promoter is delivered to cells throughout the CNS using an adeno-associated virus (AAV) vector injected into the cerebrospinal fluid (CSF). ${ }^{10}$ Preclinical studies in naturally occurring canine and feline models of MPS I demonstrated that a single injection of an AAV serotype 9 (AAV9) vector into the cisterna magna can achieve high levels of IDUA activity in the CSF within 1 week of administration, leading to resolution of storage lesions throughout the brain. ${ }^{11-13}$ Pharmacology-toxicology studies conducted in adult nonhuman primates demonstrated that the procedure was generally well tolerated but could lead to moderate subclinical lesions in a population of sensory neurons located in the dorsal root ganglia (DRG) with secondary axonopathy. ${ }^{10}$ Because the MPS I patients who could benefit the most from CNSdirected gene therapy are children, this study sought to explore the safety and efficacy of a similar intrathecal (i.t.) cervical delivery of AAV9 encoding human IDUA (hIDUA) to 1-month-old rhesus monkeys. Four animals received AAV9.CB7.CI.hIDUAco $\left(3 \times 10^{12}\right.$ genome copies [GC]/kg) by suboccipital injection (cranial to the $\mathrm{C} 1$ vertebra) at 1 month of age. Two of the animals were previously tolerized to hIDUA at birth by intravenous (i.v.) injection of an AAV8 vector expressing hIDUA from a liver-specific promoter, as previously reported (design and preliminary results published ${ }^{12}$ ).

The study demonstrates safe and sustained expression of hIDUA for almost 4 years post delivery and the absence of sensory neuron lesions, suggesting that early intervention is desirable for treating the neurologic manifestation of MPS I.

\section{METHODS \\ Animals}

All animal procedures conformed to the requirements of the Animal Welfare Act and were approved by the Institutional Animal Care and Use Committee in the AAALAC-accredited animal care program at the University of California, Davis. Normally cycling adult female rhesus monkeys (Macaca mulatta; $N=4$ ) with a history of prior pregnancy were bred and identified as pregnant according to established methods. ${ }^{14}$ Dams that were included in this study were prescreened to ensure that they were seronegative for AAV antibodies. All animals were sonographically monitored during gestation to confirm normal fetal growth and development. ${ }^{14}$ Newborns were delivered by cesarean section at term $(160 \pm 2$ days of gestation) according to established protocols. ${ }^{15}$ Newborns were placed in incubators following delivery and were nursery reared, then moved into juvenile housing for the remainder of the study. The selected AAV8 vector was administered i.v. to newborns on the day of birth. Infant health, food intake, and body weight were recorded daily in the nursery, and then weight was monitored routinely (depending on age) and according to established protocols. At 1 month of age, infants were sedated with an intramuscular (i.m.) injection of telazol $(5-8 \mathrm{mg} / \mathrm{kg})$ in preparation for CSF collection ( $\sim 0.5 \mathrm{~mL}$ ), followed by a suboccipital i.t. injection (cranial to the $\mathrm{C} 1$ vertebra; $\sim 0.5 \mathrm{~mL}$ volume; injected after collecting CSF). All procedures were performed under aseptic conditions and according to established protocols. CSF was subsequently collected at monthly or annual time points (up to 3 years and 9 months after vector administration) under telazol sedation. Blood samples were collected at birth on a weekly, monthly, or annual schedule ( $\sim 3-10 \mathrm{~mL}$ from a peripheral vessel) for complete blood counts (CBCs), clinical chemistry panels, and to obtain serum and plasma for assays (handheld in the nursery or ketamine sedation thereafter; $10-30 \mathrm{mg} / \mathrm{kg}$ i.m.). All CBCs, chemistry panels, clotting panels (at endpoint only), and CSF evaluations were performed in the Primate Center Clinical Laboratory according to established methods.

\section{Vectors}

The test article consisted of an AAV9 capsid packaging an expression construct with a hybrid promoter, which contained the cytomegalovirus enhancer coupled with a chicken beta actin promoter (CB7), a chicken beta actin intron, a codon-optimized hIDUA transgene, and a rabbit beta-globulin polyadenylation signal. ${ }^{10}$ The AAV8 vectors contained the same expression cassette downstream of the thyroid hormone binding globulin promoter or the human factor IX under the liver-specific promoter (LSP). The expression constructs were flanked by AAV2 inverted terminal repeats. The vectors were produced by triple transfecting adherent HEK293 cells. Vectors were purified from supernatant using an iodixanol gradient, as previously described. ${ }^{16}$ 
Vector titer by TaqMan polymerase chain reaction (PCR) was $6.937 \times 10^{12} \mathrm{GC} / \mathrm{mL}$ (AAV9.hIDUA), $4.75 \times 10^{13} \mathrm{GC} / \mathrm{mL}$ (AAV8.hIDUA), and $5.09 \times 10^{12}$ GC/mL (AAV8.hFIX). The purity of the capsid proteins was confirmed to be $99 \%$ (AAV9) and $100 \%$ (AAV8) using sodium dodecyl sulfate polyacrylamide gel electrophoresis analysis. The limulus amebocyte lysate test for endotoxin was negative (sensitivity $1.0 \mathrm{EU} / \mathrm{mL}$ ).

\section{Immunology}

At the final time point, peripheral blood T-cell responses were measured against hIDUA and the AAV9 and AAV8 capsids using an interferon gamma (IFN- $\gamma$ ) enzyme-linked immunospot assay according to previously published methods. ${ }^{17}$ Peptide libraries specific for the AAV capsids and the hIDUA transgene were used. The AAV8 and AAV9 capsid as well as the hIDUA transgene peptide libraries were each grouped into three peptide pools (pools A-C) for stimulation in the assay. The criteria for a positive response was $>55$ spot-forming units per $10^{6}$ lymphocytes and three times the medium negative control upon no stimulation. A medium negative control (dimethyl sulfoxide) that was higher than 55 spot-forming units did not meet quality-control specifications and was considered to be an unacceptably high level of background. Antibodies to hIDUA were measured in serum (1:1,000 sample dilution) and CSF (1:20 sample dilution), as previously described, ${ }^{12}$ using recombinant hIDUA (Aldurazyme; BioMarin/Genzyme LLC, San Rafael, CA) to coat the plates.

\section{IDUA enzyme activity}

CSF samples were placed on ice, and then frozen and stored at $\leq-80^{\circ} \mathrm{C}$ until analysis. IDUA activity was measured on black 96 -well plates by incubating $10 \mu \mathrm{L}$ undiluted sample with $50 \mu \mathrm{L} 500 \mu \mathrm{mol} / \mathrm{L} 4$ MU $\alpha$-L-iduronide (cat. \#44076; Glycosynth, Warrington, United Kingdom) in assay buffer $(0.15 \mathrm{~mol} / \mathrm{L} \mathrm{NaCl}, 0.05 \%$ Triton-X100, $0.1 \mathrm{~mol} / \mathrm{L}$ sodium acetate, $\mathrm{pH} 3.58$ ) at $37^{\circ} \mathrm{C}$ for $3 \mathrm{~h}$. The reaction was stopped by adding $150 \mu \mathrm{L} 300 \mathrm{mmol} / \mathrm{L}$ glycine, $200 \mathrm{mmol} / \mathrm{L}$ sodium citrate, $\mathrm{pH} 10.9$. The liberated $4 \mathrm{MU}$ was quantified by comparing fluorescence to standard dilutions of $4 \mathrm{MU}$ (excitation $366 \mathrm{~nm}$, emission $446 \mathrm{~nm}$ ). Units are given as nmol $4 \mathrm{MU}$ liberated per $3 \mathrm{~h}$ per milliliter of CSF. Normal values were obtained for endogenous rhesus IDUA activity from the CSF of 26 naïve rhesus monkeys (4 pre-injection and 22 unrelated to this study), including infants $(n=4)$, juveniles $(n=17)$, and young adults $(n=5)$.

\section{Tissue harvest and biodistribution}

Tissues were collected for vector biodistribution and immediately frozen in liquid nitrogen. For brain samples, sections of the frontal, temporal, and occipital cortices as well as from the hippocampus, medulla, and the left hemisphere's cerebellum were collected. Small cross-sections of the spinal cord (cervical, thoracic, and lumbar) and DRG from each spinal segment were also obtained. Samples were stored at $\leq-80^{\circ} \mathrm{C}$ until the time of analysis. DNA was extracted from tissues using QIAamp Mini Extraction kits (cat. \#51306; Qiagen, Germantown, MD). Biodistribution analysis was performed by TaqMan quantitative PCR (qPCR) targeting the vector poly-adenylation $\mathrm{rBG}$ signal sequence.

\section{Histology}

Tissues were fixed in formalin, paraffin embedded, sectioned, and stained with hematoxylin and eosin (H\&E) according to standard protocols. Tissues were histologically evaluated by a boardcertified veterinary anatomic pathologist (E.L.B.). For immunohistochemistry (IHC), the number of IDUA-positive cells was assessed using ImageJ (National Institutes of Health, Bethesda, MD) on images that were acquired using an Aperio Versa (Leica Biosystems, Buffalo Grove, IL) slide scanner. For each region, the results were expressed as a percentage of the number of transgene-positive neurons compared to the total number of the corresponding neuron type in that area. Measurements were obtained from the frontal cortex (two sections, 15 fields per section), mainly in layers III$\mathrm{V}$, to focus on the pyramidal cells. For the hippocampus, at least five fields were considered in the CA2/CA3 area. In the cerebellum, transgenepositive cells were essentially Purkinje cells; cells were counted in at least five fields per animal. The percentage of IDUA-positive motor neurons was calculated from one section for each level of the spinal cord (cervical, thoracic, and lumbar). For the DRGs, the percentage of transgene-positive cells was assessed at the cervical, thoracic, and lumbar levels in up to four whole DRGs per segment.

\section{RESULTS}

\section{Study design}

This study was designed to evaluate the longterm safety and pharmacology of an AAV9 vector encoding a codon-optimized version of hIDUA after i.t. cervical administration in 1-month-old infant rhesus monkeys. Because a vector expressing a human transgene was used, an immune response was expected against the species-specific IDUA. Considering that an antibody response to 
the human enzyme in rhesus would obscure a longitudinal assessment of expression during the in-life phase of the study, an attempt was made to induce tolerance to the hIDUA enzyme in a subset of animals (2/4). This was accomplished by delivering an i.v. dose of an AAV8 vector expressing hIDUA from a liver-specific promoter on the day of birth (AAV8.TBG.PI.hIDUAco, $1 \times 10^{12} \mathrm{GC} / \mathrm{kg}$ ). The study included four animals (Supplementary Table S1), of which half were tolerized. All animals were monitored until 3 years and 9 months of age. To control for any potential procedural effects and exposure to the AAV8 vector, the two animals in the non-tolerized group received a similar vector at birth encoding an irrelevant protein (AAV8.LSP $\left.. \mathrm{hFIXco}, 1 \times 10^{12} \mathrm{GC} / \mathrm{kg}\right)$. One month later, all animals received AAV9.CB7.CI.hIDUAco at a dose of $3 \times 10^{12} \mathrm{GC} / \mathrm{kg}$ by suboccipital (above $\mathrm{C} 1$ ) injection. A previous study reported partial results showing successful anti-hIDUA humoral response prevention for up to 2 months post injection. ${ }^{12}$

\section{Intrathecal gene transfer to infant rhesus monkeys results in sustained hIDUA expression and was well tolerated during the in-life phase of the study}

No adverse events were found during the administration period, and all animals recovered uneventfully from sedation. All animals maintained a normal growth trajectory throughout the study until the scheduled terminal endpoint (Fig. 1). A neurological examination, including general gait and locomotor activity, eye positioning, head movement, patellar reflex, foot grip, and pupillary reflex, 3 years post vector administration was unremarkable in all animals. After i.t. AAV9.hIDUA administration at 1 month of age, the CBCs and chemistry panels conducted weekly until 9 months of age and then approximately monthly thereafter were overall unremarkable and within normal limits. All parameters were comparable between animals and within the range of normal variability for this species and age group (Supplementary Tables S2-S9). These results demonstrate that the vector was well tolerated in all animals irrespective of tolerization. CBCs and chemistry panels performed prior to AAV9 administration and 2 weeks post-AAV8 newborn i.v. administration showed modestly elevated platelet counts which were within the normative range based on historical control data for rhesus monkeys in this age group and delivered by cesarean section and raised in a nursery (Dr. Tarantal, pers. commun.). Coagulation times (thromboplastin time and activated partial thromboplastin time) measured at the time of tissue harvest were within normal limits (Supplementary Table S10). Some CSF samples collected at tissue harvest were contaminated with peripheral blood, precluding meaningful interpretation (Supplementary Table S11).

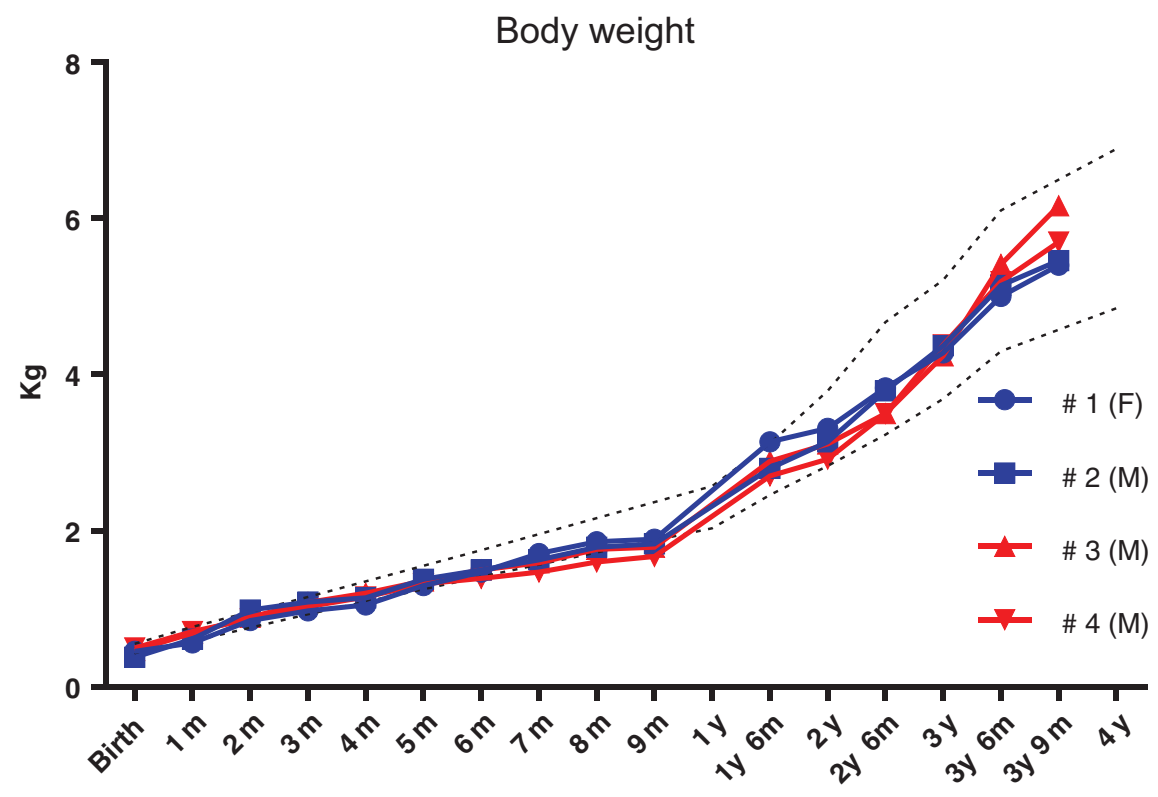

Figure 1. Body-weight gain in rhesus monkeys after a single AAV9.hIDUA intrathecal injection at 4 weeks of age. Body weight was monitored regularly and compared to historical controls. Dashed lines represent the average body weight $\pm 2 S D$ from historical controls. F, female; M, male; AAV9, adeno-associated virus serotype 9; hIDUA, human $\alpha$-L-iduronidase; $S D$, standard deviation. 
The levels of hIDUA secreted into the CSF were measured as an indicator of efficient gene transfer to the CNS. CSF IDUA activity levels increased from 2.5- to 13.5-fold over baseline in all animals 1 week following AAV9.hIDUA i.t. administration. The two animals that were previously tolerized to hIDUA achieved peak values approximately 2 months post injection (16- to 40-fold over baseline levels) and maintained supra-baseline CSF en- zyme activity until the end of the study, 3 years and 8 months post gene transfer (Fig. 2A and Supplementary Table S12). In non-tolerized animals, CSF hIDUA levels returned to baseline between 1 (\#3) and 4 months (\#4) post injection. Enzyme activity was inversely correlated with the presence of anti-hIDUA antibodies in the CSF (Fig. 2B). A sustained anti-hIDUA humoral response was observed throughout the study in the
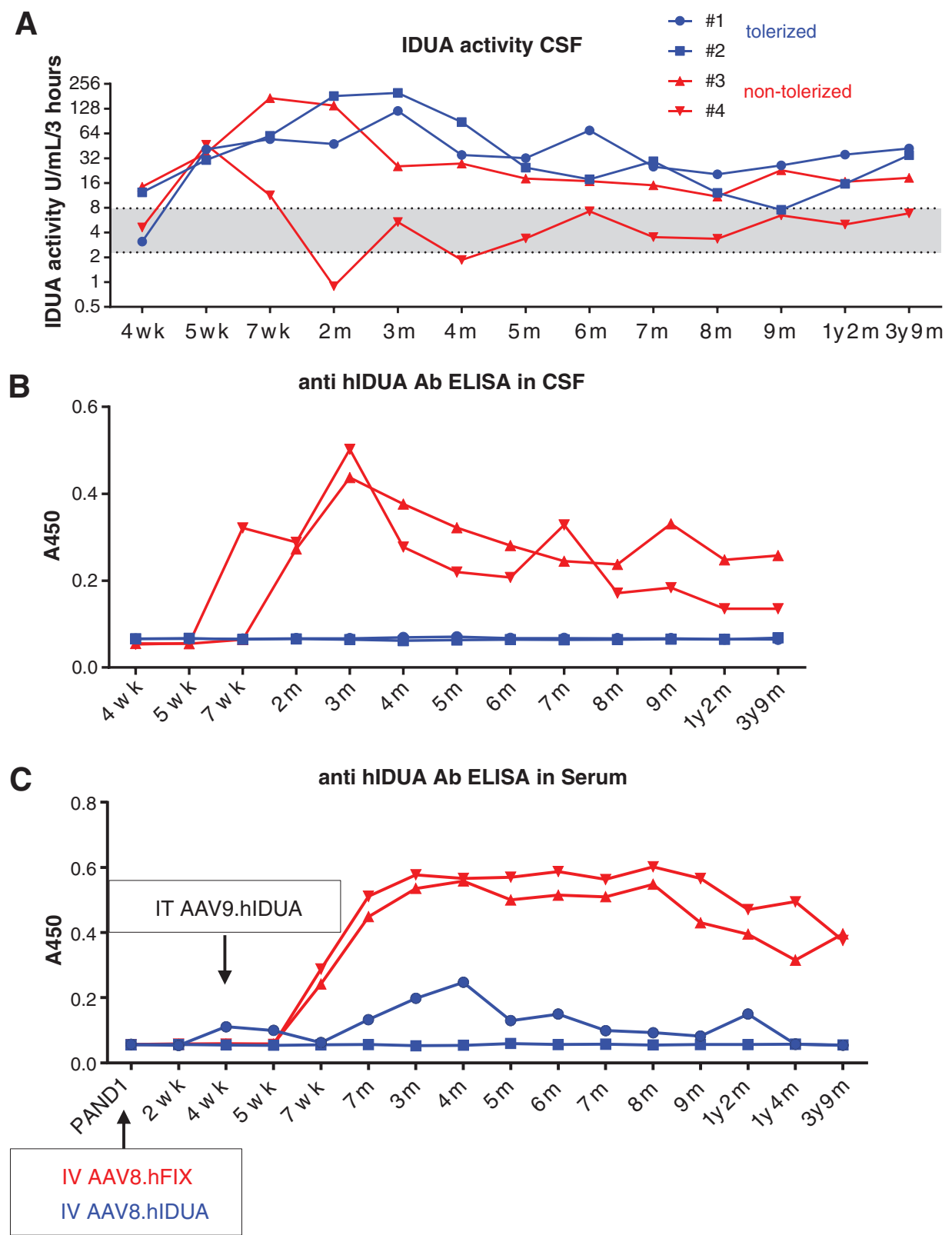

Figure 2. Sustained increased CSF IDUA activity in tolerized rhesus monkeys after a single AAV9.hIDUA intrathecal (i.t.) injection at 4 weeks of age. (A) A 4methylumbelliferone-based artificial substrate assay was used to measure IDUA activity in CSF samples from monkeys aged 4 weeks to 3 years and 9 months. Results are expressed as nanomoles of fluorescent product released per milliliter every $3 \mathrm{~h}$ (the detection limit was $0.1 \mathrm{IU} / \mathrm{mL} / 3 \mathrm{~h}$ ). The gray area between the dotted lines represents the average $\pm 1 S D$ of IDUA activity obtained at baseline in the CSF of 26 rhesus monkeys, including the pre-vector samples from infants $(N=4)$ and juveniles/adults unrelated to the study $(N=22)$. (B) ELISA measuring anti-hIDUA antibody levels in CSF (dilution 1:20). (C) ELISA measuring antihIDUA antibody levels in serum (dilution 1:1,000). CSF, cerebrospinal fluid; ELISA, enzyme-linked immunosorbent assay. 
serum of non-tolerized animals at a dilution level of $1: 1,000$. No antibody could be detected at this dilution in one tolerized animal, and a transient lowlevel response at approximately 3 months and at 1 year post injection was measured in the other tolerized animal (\#1; Fig. 2C). Peripheral blood T-cell responses against AAV8 and AAV9 capsids were negative in all animals at tissue harvest. One tolerized animal (\#1) had a low-level IFN- $\gamma$ response against one of the hIDUA peptide pools (Supplementary Table S13).

Serum hIDUA levels, from liver transduction, were transiently elevated in all animals then returned to baseline levels by 4 months of age, including in tolerized animals, likely due to loss of vector genomes in the growing liver of young animals (not shown).

\section{Analysis of tissues at harvest revealed persistent transgene expression without detectable toxicity}

Tissues from the CNS and peripheral nervous system (PNS) were analyzed for transgene genomes by PCR and for hIDUA expression by IHC. Vector genome copies were detected in all CNS and PNS tissues analyzed (Table 1 and Supplementary Fig. S1). The levels were low: 0.001-0.08 GC/diploid genome (dg) in the brain, $0.0006-0.06 \mathrm{GC} / \mathrm{dg}$ in the spinal cord, and 0.0002-0.005 GC/dg in the DRG (limit of detection $0.0001 \mathrm{GC} / \mathrm{dg}=20 \mathrm{GC}$ per microgram of DNA). There were no apparent differences between the tolerized and non-tolerized groups. However, no conclusions can be drawn due to small sample size ( $N=2$ per group) and variability inherent to sampling-induced bias when extracting DNA from small-sized tissue fragments. Furthermore, the prevalence of genomes in tissues represents an underestimate of gene transfer to neurons, as DNA is derived from all cells in the sample.

Table 1. Vector biodistribution in the CNS and PNS

\begin{tabular}{|c|c|c|c|c|}
\hline \multirow[b]{2}{*}{$G C / d g$} & \multicolumn{2}{|c|}{ Tolerized } & \multicolumn{2}{|c|}{ Non-tolerized } \\
\hline & $\# 1$ & \#2 & $\# 3$ & $\# 4$ \\
\hline Frontal cortex & 0.08 & 0.006 & 0.002 & 0.02 \\
\hline Temporal cortex & 0.02 & 0.006 & 0.006 & 0.003 \\
\hline Hippocampus & 0.01 & 0.009 & 0.02 & 0.001 \\
\hline Medulla & 0.008 & 0.007 & 0.001 & 0.008 \\
\hline Cerebellum & 0.05 & 0.002 & 0.001 & 0.01 \\
\hline Cervical spinal cord & 0.007 & 0.007 & 0.06 & 0.002 \\
\hline Thoracic spinal cord & 0.002 & 0.001 & 0.003 & 0.009 \\
\hline Lumbar spinal. cord & 0.001 & 0.0006 & 0.003 & 0.004 \\
\hline Cervical DRG & 0.002 & 0.005 & 0.0007 & 0.0002 \\
\hline Thoracic DRG & 0.002 & 0.009 & 0.003 & 0.0003 \\
\hline Lumbar DRG & 0.004 & 0.05 & 0.004 & 0.0009 \\
\hline
\end{tabular}

CNS, central nervous system; PNS, peripheral nervous system; GC/dg, genome copies per diploid genome (limit of detection $0.0001 \mathrm{GC} / \mathrm{dg}=20 \mathrm{GC}$ ) $\mu \mathrm{g}$ DNA); DRG, dorsal root ganglion.
IHC revealed expression of hIDUA above the endogenous rhesus enzyme throughout the CNS and PNS in all animals at the tissue harvest time point (Fig. 3A-G and Table 2). The most robust transduction was observed in the cerebral cortex (6-29\% of frontal cortex pyramidal neurons), hippocampus (2-13\% of neurons in layers CA2-CA3), cerebellum (8-22\% of Purkinje cells), spinal cord (6-26\% of motor neurons), and DRG $(6-23 \%$ of sensory neurons).

A board-certified veterinary pathologist analyzed a comprehensive selection of CNS, PNS, and peripheral organ tissues that were embedded in paraffin, sectioned, and stained with H\&E. All the organs were within normal limits or presented incidental background lesions unrelated to gene transfer at birth or 1 month of age. Specifically, regions that we previously identified as potential targets for toxicity after administration of pantropic vectors (namely, the DRG, spinal cord, and peripheral nerves ${ }^{10,18,19}$ ) were all unremarkable (Fig. 4A-F).

\section{DISCUSSION}

It was previously reported that both i.v. and i.t. administration of pantropic AAVs encoding various transgenes caused an asymptomatic injury to sensory neurons within the DRG, with secondary axonal degeneration in the dorsal spinal cord and peripheral nerves. ${ }^{10,18-20}$ This toxicity was observed in juvenile and adult rhesus monkeys after i.t. administration and in 7- and 30-day-old piglets after i.v. administration. The study reported herein demonstrates an absence of such toxicity 3 years and 8 months after i.t. administration of the same vector that previously caused toxicity in adult rhesus monkeys. ${ }^{10}$ To allow dose comparison, the vector lot used in the present study was re-titered using the same method as the vector lot used in adults (Taqman optimized $\mathrm{qPCR}^{21}$ ); the dose was converted to genome copies per gram of brain to account for brain growth in adults versus infant rhesus monkeys. Adult animals received $1.1 \times 10^{11}$ GC/g brain and infants received $1 \times 10^{11} \mathrm{GC} / \mathrm{g}$ brain. Some factors differ between these studies: the age at injection (1-month-old infants vs. adult rhesus monkeys), the time elapsed after intrathecal gene transfer (3 years and 8 months vs. 90180 days), and the source and location of the animals during the study. Of note, the DRG and axonopathy findings observed previously in adult rhesus were subclinical without any detectable impact based on neurological examinations. Therefore, the lack of findings on histopathology at tissue harvest does 

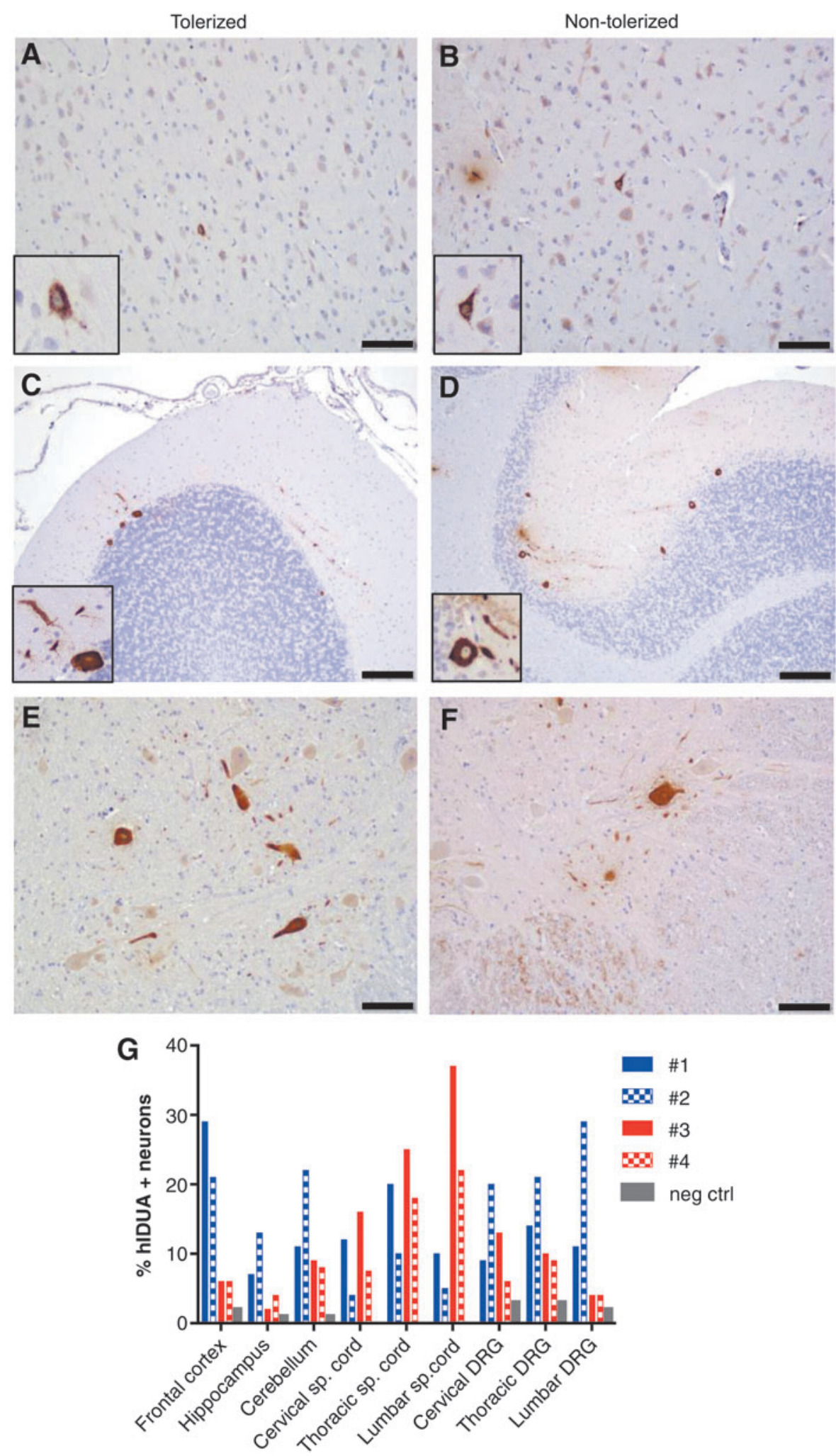

Figure 3. Long-term expression of hIDUA in neurons. Immunostaining of hIDUA 3 years and 8 months post AAV9.hIDUA i.t. injection. Representative stains from the frontal cortex (A and B), cerebellum (C and D), and cervical spinal cord (E and F) are shown. Scale bars: (A, B, E, and F) $100 \mu \mathrm{m} ;(\mathbf{C}$ and $\mathbf{D}) 200 \mu \mathrm{m}$. (G) Quantification of hIDUA-positive neurons (\% of total two neurons) in deep layers of the frontal cortex, hippocampus layers CA2-CA3, Purkinje cell layer in the cerebellum, cervical, thoracic, lumbar spinal cord anterior horns, cervical, thoracic, and lumbar DRG. Negative control=untreated rhesus monkey tissues stained with the same hIDUA antibody. 
Table 2. Quantification of hIDUA expression in neurons stained by immunohistochemistry

\begin{tabular}{lrrrrr}
\hline & \multicolumn{2}{c}{ Tolerized } & & \multicolumn{2}{c}{ Non-tolerized } \\
\cline { 2 - 3 } \cline { 5 - 5 } \% hIDUA + neurons & $\# 1$ & $\# 2$ & & $\# 3$ & $\# 4$ \\
\hline Frontal cortex & 29 & 21 & 6 & 6 \\
Hippocampus & 7 & 13 & 2 & 4 \\
Cerebellum & 11 & 22 & 9 & 8 \\
Cervical spinal cord & 12 & 4 & 16 & 7.5 \\
Thoracic spinal cord & 20 & 10 & 25 & 18 \\
Lumbar spinal cord & 10 & 5 & 37 & 22 \\
Cervical DRG & 9 & 20 & 13 & 6 \\
Thoracic DRG & 14 & 21 & 10 & 9 \\
Lumbar DRG & 11 & 29 & 4 & 4 \\
\hline
\end{tabular}

$\%$ neurons $=$ mean percentage of hIDUA-positive neurons over total number of same-type neurons in frontal cortex pyramidal cells ( 15 fields); the hippocampus CA2-CA3 region (5 fields); the Purkinje cell layer of cerebellum ( 5 fields); the anterior horns of spinal cord sections (1 section per segment); and the DRG (up to 4 DRG per segment).

hIDUA, human $\alpha$-L-iduronidase.

not necessarily imply that the injury may not have been present in earlier post-administration time points. The results suggest that infant rhesus may be less prone to injury of DRG neurons, or that repair may be more efficient in infants and/or could potentially occur after a period not captured in the 180-day study ( 6 months post transfer). In primary sensory neurons, the cell body resides in the PNS (DRG) and extends two branches from a unipolar axon: a peripheral axon that can regenerate when injured and a central axon that enters the dorsal spinal cord and does not regenerate. ${ }^{22}$ Several groups have demonstrated that DRG neurons have increased regenerative capacity compared to the CNS neurons, especially during the postnatal period. ${ }^{23-25}$ Under certain circumstances, dorsal column CNS axons can efficiently regenerate, provided that the cell body remains viable and reactivates the dormant growth ability that is characteristic of immature neurons. ${ }^{26}$ This regeneration, possibly enhanced by the age of the animals, may explain the absence of axonopathy almost 4 years after gene transfer to infant rhesus monkeys. It is also possible that infants are less susceptible to the potential for DRG injury, possibly due to lower inflammatory responses following innate immune system activation (reviewed in Simon et $a l .{ }^{27}$ and Levy ${ }^{28}$ ).

The longest persistence to date of transgene expression following intrathecal gene therapy is reported. Neuronal expression 15 years after direct intracerebral gene therapy has been demonstrated in a nonhuman primate model of Parkinson disease ${ }^{29}$ as well as long-term secretion of proteins from muscle and/or liver expression in adult animals. ${ }^{30-33}$ Other studies that have focused on gene transfer in fetuses and newborns have shown longterm gene expression in muscles or the heart for several years without any evidence of adverse findings. ${ }^{34-38}$ By contrast, when AAV vectors are administered to the liver of newborns, transgene expression can be significantly reduced over time, ${ }^{39}$ making liver-directed gene therapy potentially challenging for the treatment of pediatric diseases. In this study, CNS and PNS vector ge-
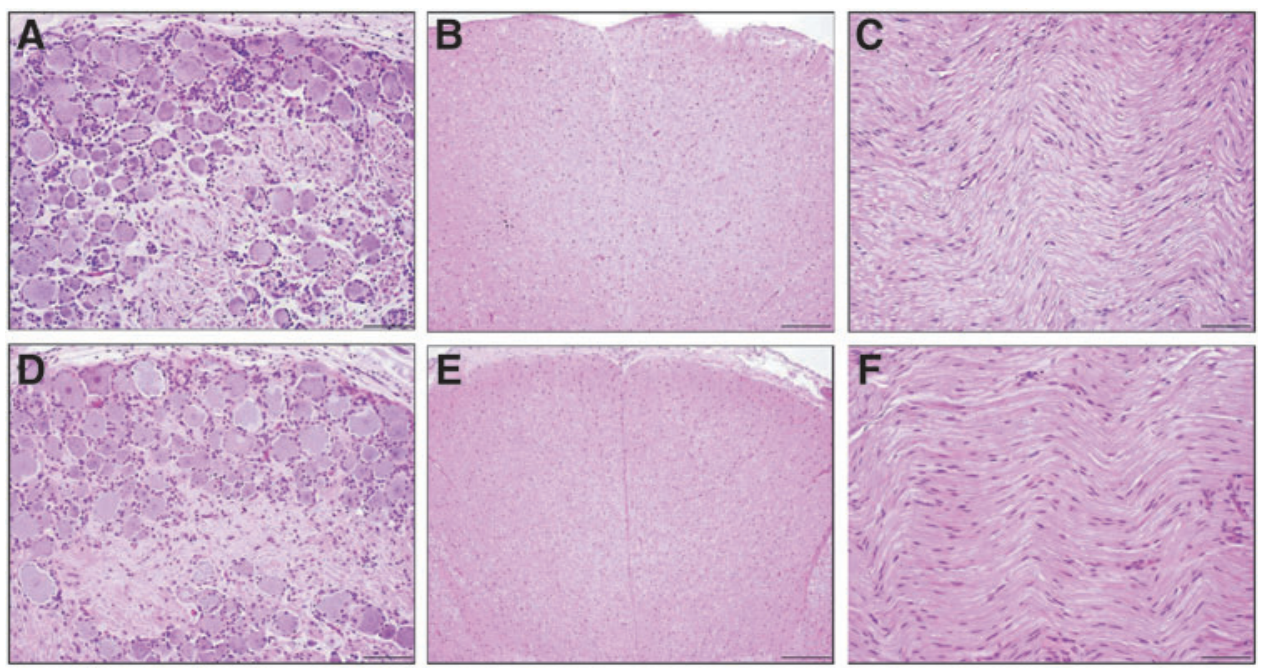

Figure 4. Representative histology of DRG, spinal cord axons, and peripheral nerves. Microscopic images of central and peripheral nervous system tissues of rhesus monkeys administered AAV9 vector expressing human IDUA via intrathecal cervical injection at 4 weeks of age. There were no test article-related microscopic findings in either the tolerized or non-tolerized cohort. The images are representative of the central and peripheral nervous system tissues from each cohort. The cervical DRG ( $\mathbf{A}$ and $\mathbf{D})$, thoracic spinal cord (B and $\mathbf{E})$, and sciatic nerve (C and $\mathbf{F}$ ) were microscopically normal in all animals. Hematoxylin and eosin staining. Scale bars: (A, C, D, and F) $100 \mu \mathrm{m}$; (B and E) $200 \mu \mathrm{m}$. 
nome copies were 10 - to 100 -fold lower than those achieved when injecting adult animals, ${ }^{10}$ suggesting that growth-related transgene dilution also occurs in nervous tissues. However, based on hIDUA staining, $6-29 \%$ of cortical pyramidal neurons, 2$13 \%$ of hippocampal pyramidal neurons, $8-22 \%$ of Purkinje cells, $5-37 \%$ of motor neurons, and $4-20 \%$ of DRG neurons expressed the transgene at tissue harvest almost 4 years post gene delivery. In the context of lysosomal storage disease in which crosscorrection is expected, sustained expression of therapeutically relevant levels of enzymes can therefore be achieved from neuronal transduction after a single intrathecal AAV administration to infants. Moreover, this strategy was well tolerated and did not lead to any adverse developmental findings, warranting clinical development for a variety of pediatric neuropathic lysosomal storage disorders such as Hurler disease.

The formation of antibodies to hIDUA led to a decrease and loss of detectable activity in the CSF of animals that were not previously tolerized to the human transgene at birth, as previously reported. ${ }^{12}$ Both tolerized and non-tolerized animals, however, had comparable levels of hIDUAstained neurons in CNS and PNS tissues. This immune response may have been enhanced by the use of a xenoantigen, as was previously reported in the context of other storage diseases. ${ }^{40}$ The impact of circulating antibodies on the efficacy of gene therapy is unknown. In MPS I mice, antibodies against recombinant human IDUA enhance uptake of the enzyme by macrophages and target it to reticuloendothelial cells in the liver. ${ }^{41}$ In feline and canine models of MPS I, the antibodies elicited after intrathecal AAV9.IDUA gene therapy resulted in a decrease of enzyme activity in the fluids of some animals; biomarkers of efficacy demonstrated a persistent biochemical response to gene transfer in cats ${ }^{11}$ and decreased clearance of storage in dogs. ${ }^{12,13}$ Importantly, the presence of antibodies did not cause any adverse events in these studies. Finally, no T cells to the AAV capsids were detected in any of the rhesus monkeys or, importantly, any apparent sequelae of destructive $\mathrm{T}$ cells.

In conclusion, this study focused on intrathecal AAV9-delivered hIDUA in infant rhesus monkeys via cervical administration and demonstrated safety and long-term potency, supporting clinical development for early-onset severe forms of neuropathic storage diseases such as MPS I (Hurler disease). The sustained long-term expression of the enzyme and the absence of sensory neuron toxicity, contrary to prior observations when treating adult rhesus monkeys, suggest that the optimal therapeutic window may be at or within months after birth. Early intervention is also preferable to treat individuals prior to irreversible neurodegeneration.

\section{ACKNOWLEDGMENTS}

This study was funded by REGENXBIO and the Orphan Disease Center Pilot Grant Program for Improved Therapies of MPS I and was supported by the NHLBI Center for Fetal Monkey Gene Transfer for Heart, Lung, and Blood Diseases (NIH grant HL085794) and the Primate Center base operating grant (NIH grant OD011107). The authors would like to acknowledge the University of Pennsylvania Gene Therapy Program cores: the Penn Vector Core, Morphology Core, and Immunology Core.

\section{AUTHOR DISCLOSURE}

J.M.W. is an advisor to, holds equity in, and has a sponsored research agreement with Scout Bio and Passage Bio. He also has a sponsored research agreement with Ultragenyx, Biogen, Janssen, Precision Biosciences, Moderna Therapeutics, and Amicus Therapeutics, which are licensees of Penn technology. He is an inventor on patents that have been licensed to various biopharmaceutical companies. No competing financial interests exist for the remaining authors.

\section{SUPPLEMENTARY MATERIAL}

Supplementary Table S1

Supplementary Table S2

Supplementary Table S3

Supplementary Table S4

Supplementary Table S5

Supplementary Table S6

Supplementary Table S7

Supplementary Table S8

Supplementary Table S9

Supplementary Table S10

Supplementary Table S11

Supplementary Table S12

Supplementary Table S13

Supplementary Figure S1 


\section{REFERENCES}

1. Beck $M$, Arn P, Giugliani R, et al. The natural history of MPS I: global perspectives from the MPS I Registry. Genet Med 2014;16:759-765.

2. Vijay S, Wraith JE. Clinical presentation and follow-up of patients with the attenuated phenotype of mucopolysaccharidosis type I. Acta Paediatr 2005;94:872-877

3. Matte U, Yogalingam G, Brooks D, et al. Identification and characterization of 13 new mutations in mucopolysaccharidosis type I patients. Mol Genet Metab 2003;78:37-43.

4. Clarke LA, Wraith JE, Beck M, et al. Long-term efficacy and safety of laronidase in the treatment of mucopolysaccharidosis I. Pediatrics 2009;123:229-240.

5. Sifuentes M, Doroshow R, Hoft R, et al. A followup study of MPS I patients treated with laronidase enzyme replacement therapy for 6 years. Mol Genet Metab 2007;90:171-180.

6. Wraith JE, Clarke LA, Beck M, et al. Enzyme replacement therapy for mucopolysaccharidosis I: a randomized, double-blinded, placebo-controlled, multinational study of recombinant human $\alpha-\mathrm{L}$ iduronidase (laronidase). J Pediatr 2004;144:581-588.

7. Wraith JE, Beck M, Lane R, et al. Enzyme replacement therapy in patients who have mucopolysaccharidosis I and are younger than 5 years: results of a multinational study of recombinant human alpha-L-iduronidase (Laronidase). Pediatrics 2007;120:E37-E46.

8. de Ru MH, Boelens JJ, Das AM, et al. Enzyme replacement therapy and/or hematopoietic stem cell transplantation at diagnosis in patients with mucopolysaccharidosis type I: results of a European consensus procedure. Orphanet J Rare Dis 2011;6:55.

9. Kennedy DW, Abkowitz JL. Kinetics of central nervous system microglial and macrophage engraftment: analysis using a transgenic bone marrow transplantation model. Blood 1997;90:986-993.

10. Hordeaux J, Hinderer C, Goode T, et al. Toxicology study of intra-cisterna magna adeno-associated virus 9 expressing human alpha-L-iduronidase in rhesus macaques. Mol Ther Methods Clin Dev 2018;10:79-88.

11. Hinderer C, Bell P, Gurda BL, et al. Intrathecal gene therapy corrects CNS pathology in a feline model of mucopolysaccharidosis I. Mol Ther 2014; 22:2018-2027.

12. Hinderer C, Bell P, Louboutin JP, et al. Neonatal systemic AAV induces tolerance to CNS gene therapy in MPS I dogs and nonhuman primates. Mol Ther 2015;23:1298-1307.

13. Hinderer C, Bell P, Louboutin JP, et al. Neonatal tolerance induction enables accurate evaluation of gene therapy for MPS I in a canine model. Mol Genet Metab 2016;119:124-130.

14. Tarantal AF. Ultrasound imaging in rhesus (Macaca mulatta) and long-tailed (Macaca fascicularis) macaques: reproductive and research applications. In: Wolfe-Coote S, ed. The Labora- tory Primate. London: Elsevier Academic Press, 2005:317-352.

15. Tarantal AF, McDonald RJ, Jimenez DF, et al. Intrapulmonary and intramyocardial gene transfer in rhesus monkeys (Macaca mulatta): safety and efficiency of HIV-1-derived lentiviral vectors for fetal gene delivery. Mol Ther 2005;12:87-98.

16. Lock M, Alvira M, Vandenberghe LH, et al. Rapid, simple, and versatile manufacturing of recombinant adeno-associated viral vectors at scale. Hum Gene Ther 2010;21:1259-1271.

17. Gao G, Wang Q, Calcedo R, et al. Adenoassociated virus-mediated gene transfer to nonhuman primate liver can elicit destructive transgene-specific $T$ cell responses. Hum Gene Ther 2009;20:930-942.

18. Hordeaux J, Hinderer C, Goode T, et al. Toxicology study of intra-cisterna magna adeno-associated virus 9 expressing iduronate-2-sulfatase in rhesus macaques. Mol Ther Methods Clin Dev 2018;10:68-78.

19. Hinderer C, Katz N, Buza EL, et al. Severe toxicity in nonhuman primates and piglets following highdose intravenous administration of an adenoassociated virus vector expressing human SMN. Hum Gene Ther 2018;29:285-298.

20. Hordeaux J, Wang 0 , Katz N, et al. The neurotropic properties of AAV-PHP.B are limited to C57BL/6J mice. Mol Ther 2018;26:664-668.

21. Lock M, Alvira MR, Chen SJ, et al. Absolute determination of single-stranded and selfcomplementary adeno-associated viral vector genome titers by droplet digital PCR. Hum Gene Ther Methods 2014;25:115-125.

22. Neumann S, Woolf CJ. Regeneration of dorsal column fibers into and beyond the lesion site following adult spinal cord injury. Neuron 1999; 23:83-91.

23. Mackenzie IR, Frick P, Grasser FA, et al. Quantitative analysis and clinico-pathological correlations of different dipeptide repeat protein pathologies in C90RF72 mutation carriers. Acta Neuropathol 2015;130:845-861.

24. Arora DK, Cosgrave AS, Howard MR, et al. Evidence of postnatal neurogenesis in dorsal root ganglion: role of nitric oxide and neuronal restrictive silencer transcription factor. J Mol Neurosci 2007;32:97-107.

25. Namaka MP, Sawchuk M, MacDonald SC, et al. Neurogenesis in postnatal mouse dorsal root ganglia. Exp Neurol 2001;172:60-69.

26. He Z, Jin Y. Intrinsic Control of Axon Regeneration. Neuron 2016;90:437-451.

27. Simon AK, Hollander GA, McMichael A. Evolution of the immune system in humans from infancy to old age. Proc Biol Sci 2015;282:20143085.

28. Levy 0 . Innate immunity of the newborn: basic mechanisms and clinical correlates. Nat Rev Immunol 2007:7:379-390.
29. Sehara Y, Fujimoto KI, Ikeguchi K, et al. Persistent expression of dopamine-synthesizing enzymes 15 years after gene transfer in a primate model of Parkinson's disease. Hum Gene Ther Clin Dev 2017:28:74-79.

30. Rivera VM, Gao GP, Grant RL, et al. Long-term pharmacologically regulated expression of erythropoietin in primates following AAV-mediated gene transfer. Blood 2005;105:1424-1430.

31. Wang L, Calcedo R, Nichols TC, et al. Sustained correction of disease in naive and AAV2-pretreated hemophilia B dogs: AAV2/8-mediated, liver-directed gene therapy. Blood 2005;105:3079-3086.

32. Herzog RW, Yang EY, Couto LB, et al. Long-term correction of canine hemophilia B by gene transfer of blood coagulation factor IX mediated by adeno-associated viral vector. Nature Medicine 1999;5:56-63.

33. Greig JA, Peng H, Ohlstein J, et al. Intramuscular injection of AAV8 in mice and macaques is associated with substantial hepatic targeting and transgene expression. PLoS One 2014;9:e112268.

34. Tarantal AF, Skarlatos SI. Center for Fetal Monkey Gene Transfer for Heart, Lung, and Blood Diseases: an $\mathrm{NHLBI}$ resource for the gene therapy community. Hum Gene Ther 2012;23:1130-1135.

35. Tarantal AF, Lee CCl, Martinez ML, et al. Systemic and persistent muscle gene expression in rhesus monkeys with a liver de-targeted adeno-associated virus vector. Hum Gene Ther 2017;28:385-391.

36. Tarantal AF, Lee CC. Long-term luciferase expression monitored by bioluminescence imaging after adeno-associated virus-mediated fetal gene delivery in rhesus monkeys (Macaca mulatta). Hum Gene Ther 2010;21:143-148.

37. Tai DS, Hu C, Lee CC, et al. Development of operational immunologic tolerance with neonata gene transfer in nonhuman primates: preliminary studies. Gene Ther 2015;22:923-930.

38. Conlon TJ, Mah CS, Pacak CA, et al. Transfer of therapeutic genes into fetal rhesus monkeys using recombinant adeno-associated type I viral vectors. Hum Gene Ther Clin Dev 2016;27:152-159.

39. Wang L, Bell P, Lin J, et al. AAV8-mediated hepatic gene transfer in infant rhesus monkeys (Macaca mulatta). Mol Ther 2011;19:20122020

40. Haurigot V, Marco S, Ribera A, et al. Whole body correction of mucopolysaccharidosis IIIA by intracerebrospinal fluid gene therapy. J Clin Invest 2013;123:3254-3271.

41. Le SQ, Kan SH, Clarke D, et al. A humoral immune response alters the distribution of enzyme replacement therapy in murine mucopolysaccharidosis type I. Mol Ther Methods Clin Dev 2018;8:42-51.

Received for publication January 16, 2019; accepted after revision April 13, 2019

Published online: April 23, 2019 